\title{
Induced Jet Decay from Flow-Channel Streams
}

Anna Pennemann*, Ruolan Yang, Gerhard Schaldach and Markus Thommes

Laboratory of Solids Process Engineering, TU Dortmund University, Dortmund, Germany

*Corresponding author email : anna.pennemann@tu-dortmund.de

\begin{abstract}
Monodisperse droplets and sprays are employed in various industries and are frequently produced via induced jet decay in the Rayleigh regime. However, these processes are limited to vertical jets exciting from capillaries and small nozzle orifices.

Investigation results into the transferability of this process to flow channel streams are presented. Experiments were performed under variation of the flow rate, viscosity, excitation frequency and tilt angle.

Monodisperse droplets were successfully produced from flow channel streams at process parameters comparable to those of the corresponding measurements employing capillaries.
\end{abstract}

\section{Keywords}

spraying, liquid jets, monodisperse droplets

\section{Introduction}

The production of small droplets is highly important for a wide range of applications, including spray drying, liquid fuel combustion and coating processes. In many of these processes, the employment of monodisperse droplets can enhance the process efficiency and product quality. [1, 2]

The production of monodisperse droplets can be performed via induced jet decay. In this method, a liquid jet fulfilling the conditions for Rayleigh-type disintegration is disturbed at a defined frequency in order to trigger the jet breakup in a controlled manner. As established by Lord Rayleigh [3], the employed frequency must correspond to a wavelength $\lambda$ larger than the jet circumference and the optimum wavelength $\lambda_{\text {opt }}$ for non-viscous, incompressible fluids can be defined as a function of the jet diameter $\mathrm{d}$ :

$$
\lambda_{\text {opt }}=4.5 d
$$

This was expanded to viscous fluids in subsequent publications to [1]

$$
\lambda_{\text {opt }}=\pi d(2+6 O h)^{1 / 2}
$$

with

$$
O h=\frac{\eta}{(\rho \sigma d)^{12}}
$$

The nozzle diameter can be substituted for the jet diameter with little error for vertical jets with

$$
B o=\frac{d_{n}{ }^{2} \rho g}{\sigma}<0.5 \text {. [1] }
$$

However, these theoretical and experimental observations focused solely on jets exciting from closed systems and typically utilize the nozzle diameter as a substitute for the jet diameter. This limits the applicability and necessitates a change in the employed capillary when varying process parameters. Therefore, the induced jet decay from a flow channel was investigated. Flow channels with semi-circular circumferences and a radius much larger than the hydraulic diameter of the fluid were used to ensure uniform conditions and to enable a description of the fluid flow based on the fluid flow on tilted planes as established by Schröder. [4] Figure 1 
demonstrates the geometry of the flow channel as well as the relation between the channel radius $R$ and the hydraulic diameter $\delta$ of the fluid.

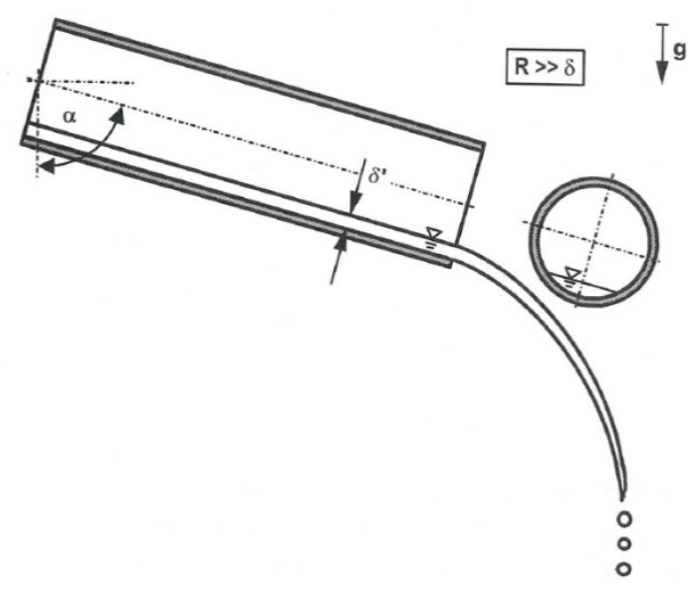

Figure 1. Schematic representation of the flow channel streams, modified from [4].

As the low volume flow rates necessary to ensure laminar flow in the flow channel setup result in Bond numbers larger than 0.5, significant jet diameter reduction can be observed in addition to the jet deformation at the flow channel edge. Thus, the experimental data shown will be presented in dependence of the employed frequencies, not in dependence of the wavelength as a function of jet diameter for both the capillary and the flow channel setup.

\section{Material and Methods}

The transfer potential of the principles and process of the induced jet decay was investigated under variation of the viscosity, volume flow rate and tilt angle. An example of the employed experimental set-up is shown for the vertical capillary in figure 2.

Glycerine-water-mixtures with viscosities of $0.001,0.01$ and 0.1 Pas were pumped at volume flow rates of 3.5, 3.7 and $3.9 \mathrm{l} / \mathrm{h}$ by a micro-gear pump. The pump was custom-built and offers pulsation-free volume flow rates of 0 up to $4.4 \mathrm{l} / \mathrm{h}$. It comprises a pump head (MZR-7205, HNPMikrosysteme, Schwerin, Germany), a Coriolis flow meter (Mini Cori-Flow, Bronkhorst, Kamen, Germany) and a micro-controller (Arduino Mega 2560, Arduino LLC, Ivrea, Italy).

Primary experiments were performed in a glass flow channel with a tilt angle of $65^{\circ}$ and an inner diameter of $20 \mathrm{~mm}$, to ensure that the boundary condition of $R \gg>\delta$ [4] was fulfilled for all volume flow rates. A vertical capillary with a nozzle diameter of $2 \mathrm{~mm}$ was used for reference purposes.

The sinusoidal disturbance signals originated from a frequency generator (Voltcraft 8202, Conrad Electronic SE, Hirschau, Germany) and were verified by an external frequency monitor (TF830,Thurlby Thandar Instruments, Huntingdon, UK) before being translated into mechanical vibrations by the vibration exciter (4809, Brüel \& Kjær, Nærum, Denmark).

For secondary experiments, nozzle and channel diameters remained constant. The respective geometries were 3D-printed from PLA-filaments to ensure identical wetting behaviour and mounted at tilt angles of $0^{\circ}, 25^{\circ}$ and $65^{\circ}$ to the vertical axis. The trailing edge was located vertically below the vibration exciter for all geometries.

The droplets formed in the jet decay were photographed with a compact camera (Nikon Z6 24-70/4 S, Sendai Nikon Corporation, Natori, Japan) with backlighting from an LED-panel and subsequently binarised and analysed in Fiji 1.53c [5,6]. 


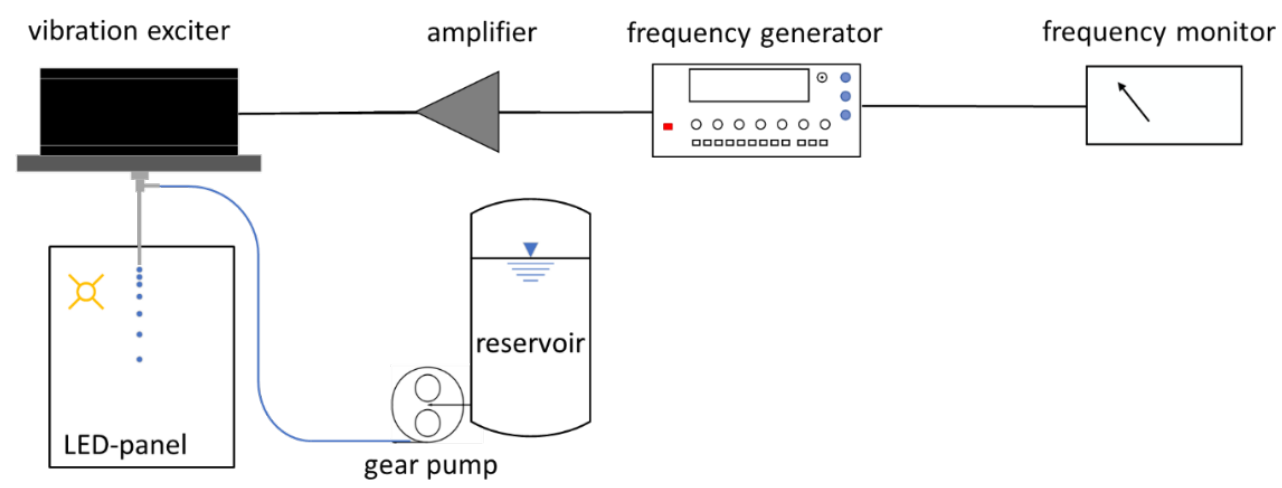

Figure 2. Experimental setup.

\section{Results and Discussion}

Induced jet decay resulting in stable droplet patterns was possible for all geometries over a large frequency range. Figure 3 shows an example of the resulting median droplet size $d_{50,3}$ in dependence of the excitation frequency as well as the expected size for ideally monodisperse droplets.

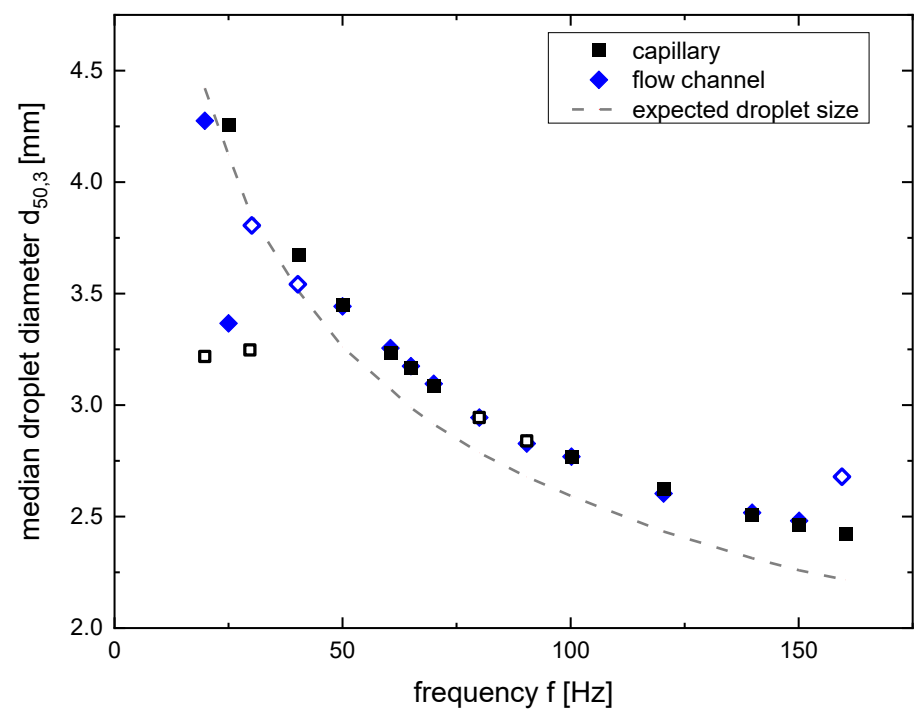

Figure 3. Median droplet size $d_{50,3}$ as a function of the excitation frequency for an angle of $\alpha=65^{\circ}$; Filled symbols indicate monodispersity according to VDI-guideline 3491 [7].

Excitation frequencies below $40 \mathrm{~Hz}$ led to periodic droplet patterns associated with long-range Rayleigh-breakup [8], as shown in figure 4, and thus to multimodal droplet size distribution functions. 

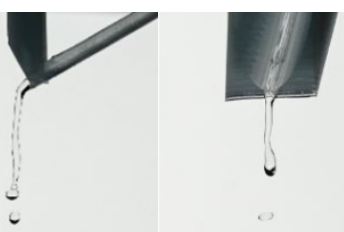

Figure 4. Jet decay with periodic droplet pattern formation for a tilt angle of $\alpha=65^{\circ}$ (left) and $\alpha=25^{\circ}$ (right) for an excitation frequency of $f=20 \mathrm{~Hz}$.

Monodisperse droplet formation was generally observed for frequencies of $50 \mathrm{~Hz}$ and upwards; exemplary images of the resulting droplet streams as well as a more detailed comparison of the droplet size distributions resulting from capillary and flow channel jets are shown in Figure 5. The resulting size distributions not only matched in regards to the median droplet diameter, but also overlapped in respect to their distribution functions.
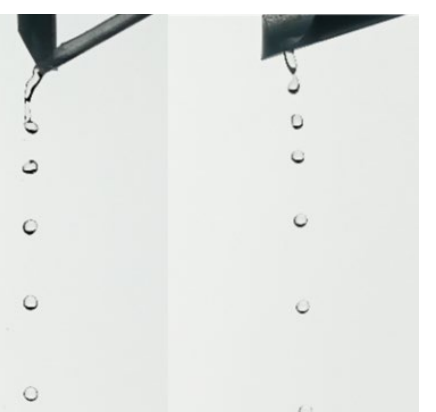

a)

b)

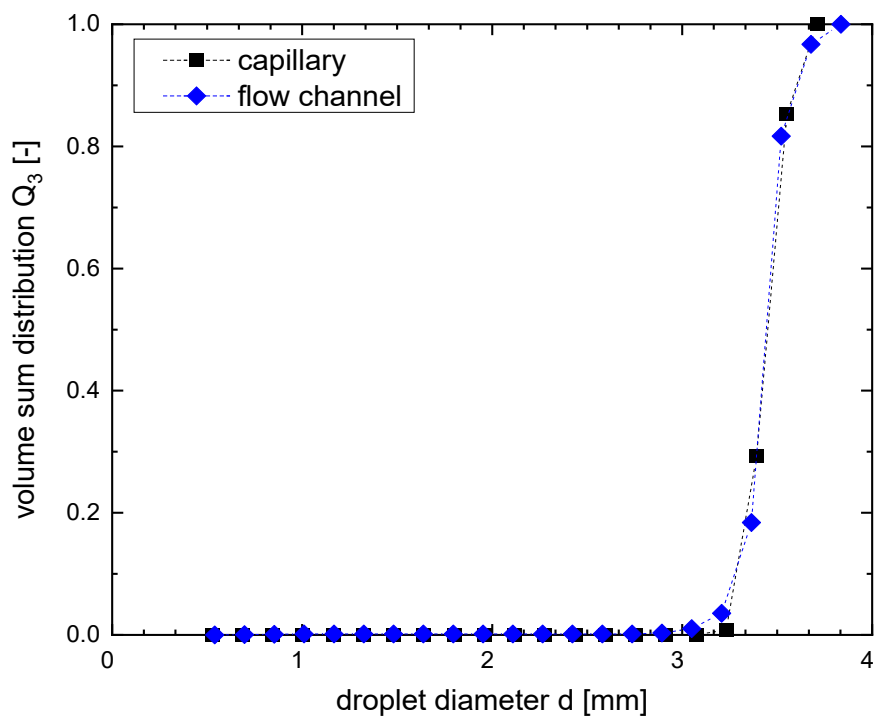

Figure 5. Monodisperse jet decay (left) from a capillary (a) in comparison to a flow channel (b) and the resulting cumulative volume distributions $Q_{3}$ (right) for a tilt angle of $\alpha=65^{\circ}$ at an excitation frequency $f=50 \mathrm{~Hz}$.

While some data points above $50 \mathrm{~Hz}$ did not fulfill the VDI-criteria for monodispersity, they still exhibited controlled and uniform droplet formation. At these data points, stable satellite drops were generated at a ratio of 1:1 with the main droplets, a phenomenon which has previously 
been described for mid-range Rayleigh-breakup [8]. This occurred mainly for the larger tilt angles at frequencies of about $80 \mathrm{~Hz}$.

All results were in accordance with the expected values and showed high levels of similarity between the different geometries.

\section{Conclusions}

The generation of monodisperse droplets by induced jet decay from flow channel streams was proven to be feasible and shows promise of a high level of transferability regarding the concept as well as the governing principles. An increase in satellite drop occurrence was observed for the flow channel jets in general and in dependence of the tilt angle for capillary jets. The causality of increased satellite droplet formation is currently unclear; potential influence factors are the velocity gradient in the flow channel, the deformation upon channel exit and the free liquid interface.

Future research will focus on the development of a predictive formula for the induced jet decay from flow channels as well as the causality of satellite drop formation.

\section{Nomenclature}

$D \quad$ droplet diameter [mm]

d jet diameter [mm]

$d_{n} \quad$ nozzle diameter [mm]

$f \quad$ frequency $[\mathrm{Hz}]$

$g$ gravitational acceleration $\left[\mathrm{m} / \mathrm{s}^{2}\right]$

$R \quad$ flow channel radius [mm]

$\dot{V} \quad$ volume flow rate [l/h]

Oh Ohnesorge number [-]

Bo Bond number [-]

$\delta \quad$ hydraulic diameter [mm]

$\eta \quad$ dynamic viscosity [Pas]

$\lambda \quad$ wavelength [mm]

$\sigma \quad$ surface tension $[\mathrm{mN} / \mathrm{m}]$

$\rho \quad$ density $\left[\mathrm{kg} / \mathrm{m}^{3}\right]$

\section{References}

[1] Walzel, P., "Spraying and Atomizing of Liquids", Ullmann's Encyclopedia of Industrial Chemistry, pp.1-30

[2] Etzold, M., Zeitmodulierte, monodisperse Tropfengenerierung zur Herstellung von Feinstsprays 2019, FAU University Press: Erlangen.

[3] Rayleigh, L., On The Instability Of Jets. Proceedings of the London Mathematical Society, 1878. s1-10(1): p. 4-13.

[4] Schröder, T., Tropfenbildung an Gerinneströmungen im Schwere- und Zentrifugalfeld. 1997: VDI-Verlag.

[5] Schindelin, J., Arganda-Carreras, I., Frise, E., Kaynig, V., Longair, M., Pietzsch, T., Preibisch, S., Rueden, C., Saalfeld, S., Schmid, B., Tinevez, J.-Y., White, D. J., Hartenstein, V., Eliceiri, K., Tomancak, P., and Cardona, A., 2012, "Fiji: an open-source platform for biological-image analysis," Nat. Methods, 9(7), pp. 676-682.

[6] Schneider, C. A., Rasband, W. S., and Eliceiri, K. W., 2012, "NIH Image to ImageJ: 25 years of image analysis," Nat. Methods, 9(7), pp. 671-675.

[7] Society of German Engineers (VDI): VDI Guideline 3491, in: VDI-Handbuch Reinhaltung der Luft, Band 4, Düsseldorf 2016

[8] Brenn, G., Die gesteuerte Sprayerzeugung für industrielle Anwendungen. 1999. 